\title{
Computer 3D controlled bacterial transports and aggregations of microbial adhered nano-components
}

\author{
Sylvain Martel • Samira Taherkhani • \\ Maryam Tabrizian • Mahmood Mohammadi • \\ Dominic de Lanauze • Ouajdi Felfoul
}

Received: 31 December 2013 / Revised: 24 March 2014 / Accepted: 15 May 2014 /Published online: 25 May 2014

(C) Springer-Verlag Berlin Heidelberg 2014

\begin{abstract}
Bacteria can be used as microcarriers for the transport of nano-components. By exploiting specific taxes such as chemotaxis, aerotaxis or phototaxis, to name but a few examples, sensory-based transports of nano-components towards or away from a specific source of chemical, oxygen, or light for instance, can be achieved. Using microbial adhesion methods, such nano-components can be attached to the surface of magnetotactic bacteria (MTB), allowing for precise magnetotaxis computer controlled transport of these nanocomponents along a planned path on a planar surface or towards a specific region within a 3D space. Here, the possibility of attaching a large number of nano-components to the surface of magnetotactic bacteria combined with the possibility of aggregating a large number of cells in a 3D space suggests the possibility of delivering and aggregating a large number of nano-components at a specific region within a relatively large 3D space using relatively low electrical energy.
\end{abstract}

Keywords Bacteria $\cdot$ Magnetotaxis $\cdot$ Nano-components · Adhesion $\cdot$ Bacterial transport

This paper is published in a Special Issue "3M-NANO 2013" organized by Prof. Dr. Sergej Fatikow and Prof. Dr. Zuobin Wang

S. Martel $(\bowtie) \cdot$ S. Taherkhani $\cdot$ M. Mohammadi $\cdot$ D. de Lanauze $\cdot$

O. Felfoul

NanoRobotics Laboratory, Department of Computer and Software

Engineering, and Institute of Biomedical Engineering, École

Polytechnique de Montréal (EPM), Campus of the Université de

Montréal, Montréal, Canada

e-mail: sylvain.martel@polymtl.ca

M. Tabrizian

Department of Biomedical Engineering, McGill University,

Montréal, Canada

\section{Introduction}

Relatively fast accurate transports of nanoscale components in a fluidic environment can be achieved from three fundamental approaches. The first approach relies on an external source to induce a displacement force on the nano-component itself. Dielectrophoresis and the induction of a magnetic pulling force are just two examples among others. In such a case, the force induced in dependent upon specific characteristics of the nano-component to be manipulated. For instance, for a soft magnetic nano-component, the induced force can be estimated as

$\vec{F}=R \cdot V_{M}(\vec{M} \cdot \nabla) \vec{B}$

where $R$ defined in the interval 0 to 1 represents the duty cycle of the pulling force being applied, i.e., the fraction of the time within a cycle where the pulling gradient responsible to induce a pulling force on the navigable agent is being applied. $V_{M}$ is the total volume of soft magnetic material (typically ferromagnetic or superparamagnetic) embedded in the nanocomponent if the whole nano-component is not magnetic, and $\boldsymbol{M}$ is the corresponding magnetization level which increases up to a saturation level with an increase of the field $\boldsymbol{B}$. In (1), the gradient operator is expressed as

$\nabla=\left[\begin{array}{lll}\frac{\partial}{\partial x} & \frac{\partial}{\partial y} & \frac{\partial}{\partial z}\end{array}\right]^{T}$.

It can be deducted from (1) that for a nanoscale component, $V_{M}$ is extremely small and as such, the pulling force induced is reduced substantially for a given magnetization level. Unless the source is extremely close to the nano-object to be 
manipulated, inducing sufficient force on such a small component for adequate transport may prove to be challenging. One strategy corresponding to the second approach would be to use a larger carrier capable of transporting nanoscale components. Such a carrier would be characterized by a larger volume of magnetic material for instance and as such, the induced pulling force from an external source would be increased as well.

In order to decrease further the level of power required from the external source, the pulling force acting on the carrier could be replaced by a torque (Eq. 3).

$\vec{T}=\vec{m} \times \vec{B}$

In (3), the magnetic moment is expressed as

$\vec{m}=V_{M} \cdot \vec{M}$.

If a torque is used, the shape of the carriers would have to be modified accordingly. Artificial swimmers that can be used as potential carriers and characterized by a helical shape have been suggested [1-3]. Such artificial swimmers mimicking the flagellar propulsion of bacteria can perform particularly well in low Reynolds hydrodynamic conditions. To achieve a displacement, a rotating field generated from an external source is used to induce a torque for propelling these miniature helical swimmers. Transport [4] and coordinating many artificial swimmers for micromanipulation $[5,6]$ are also possible.

Although the level of power required for the displacement of artificial swimmers can be significantly reduced, a source of power is still required to induce the directional torque used to propel these magnetic swimmers.

A third option is the use of self-propelled microcarriers. Natural bacteria for instance, not only have their own source of power but also swim extremely well in low Reynolds hydrodynamic conditions [7-15]. Computer controlled transport using a flagellated Magnetotactic Bacterium (MTB) [16-20] or through the coordination of a large group of MTB [21] have been investigated and demonstrated.

To achieve accurate displacements of MTB under computer control along a planned path, magnetotaxis [22-24] is exploited. Magnetotaxis displacement control is achieved by inducing a directional torque on a chain of nanoparticles known as magnetosomes that grow in the cell $[25,26]$ during cultivation. Using feedback control based on images gathered from a microscope, a directional magnetic field can be generated from a computer to maintain such MTB along a planned path. Since MTB respond to the geomagnetic field, a very low magnitude magnetic field is required to obtain adequate directional responses from the bacteria. This is quite important when the external source of power used only for directional control and not for inducing a displacement force, must be minimized.

Other taxes from stimuli generated by an external source can also be considered. Phototaxis [27] directional control [28] for instance using a light source could also be used. To eliminate any external source of power, other taxes such as aerotaxis [29-31] or chemotaxis [32-34], to name but only two examples, can also be considered. Besides directional control, techniques to stop and resume the displacement of bacteria have also been investigated [35].

Although bacteria can be used as sophisticated microcarriers capable of sensing stimuli for influencing their displacement or acting under computer control, the use of these microorganisms for transporting nano-components requires adequate methods to adhere such nano-components to the surface of the cells.

\section{Microbial adhesion methods}

In most cases, adhesion of microorganisms to various interfaces has been explained by the classical Derjaguin-LandauVerwey-Overbeek (DLVO) theory of colloid stability [36]. Several microbial adhesion methods have been investigated. Although the surface and the characteristics of the bacterial cell change with alterations in environmental conditions, the cell surfaces are usually negatively charged with varying degree of hydrophobicity. These characteristics allow us to consider them like colloidal particles where their adhesion can be studied as a physicochemical phenomenon [37]. Bacteria differ widely with respect to their hydrophobicity and electrophoretic mobility. In an aqueous medium, hydrophobicity is related to the surface Gibbs energy and the Van de Waals interactions between particles whereas electrophoretic mobility is a measure of the electrostatic interaction between particles. Therefore, these two characteristics can be exploited to attach nano-components to the cell surface [38]. For instance, hydrophobic cells adhere to a greater extent than hydrophilic cells [39]. Besides the aforementioned interactive forces, Extracellular Polymeric Substances (EPS) can also be used [40]. EPS which are secreted by microorganisms during growth consists of many organic substances such as polysaccharides, proteins, nucleic acids and lipids. From a physicochemical viewpoint, an EPS covering on a cell surface is regarded as polyelectrolytes adsorbed onto a colloidal particle. EPS significantly influence bacterial adhesion and as such, it can be used to attach nano-components.

The presence of specific antigens can also be exploited for attaching nano-components. For instance, it is known that the presence of $\mathrm{O}$-antigen affects the adhesion of Serratia marcescens cells to inert and biological surfaces [41]. Specific 
polyclonal antibodies can be developed to adhere to the surface of the cell or the nano-components can be covalently attached. Specificity for antibodies can be improved with the use of specific monoclonal antibodies where each one would adhere to a specific site at the surface of the cell. For the latter, attaching a large number of nano-components per cell would require the development of several monoclonal antibodies which may be not only time consuming but costly as well.

Such short survey indicates the possibility of attaching a large number of nano-components to each MTB and to direct them at a specific region in a $3 \mathrm{D}$ space. Such a statement is validated through preliminary experimental results that are presented in the following section.

\section{Experimental results}

A photograph of one MC-1 magnetotactic bacterium with approximately 70 liposomes (each with a diameter of approximately $170 \mathrm{~nm}$ ) is depicted in Fig. 1. In this particular case, each liposome has been synthesized to allow the encapsulation of a drug molecule. Liposomes were attached to the surface of the MC-1 cell using covalent binding through carbodiimide chemistry resulting in very strong attachments as described and characterized in [48].

Although the addition of such high number of liposomes theoretically add to the drag force acting against the thrust propelling force of the MC-1 cell, only a relatively small decrease in swimming velocities have been observed (corresponding to approximately $27 \%$ decrease in velocity). This can be explained by the relatively small size of the attached liposomes and the relatively high thrust force of each cell that can exceed $4 \mathrm{pN}$ (measured experimentally).

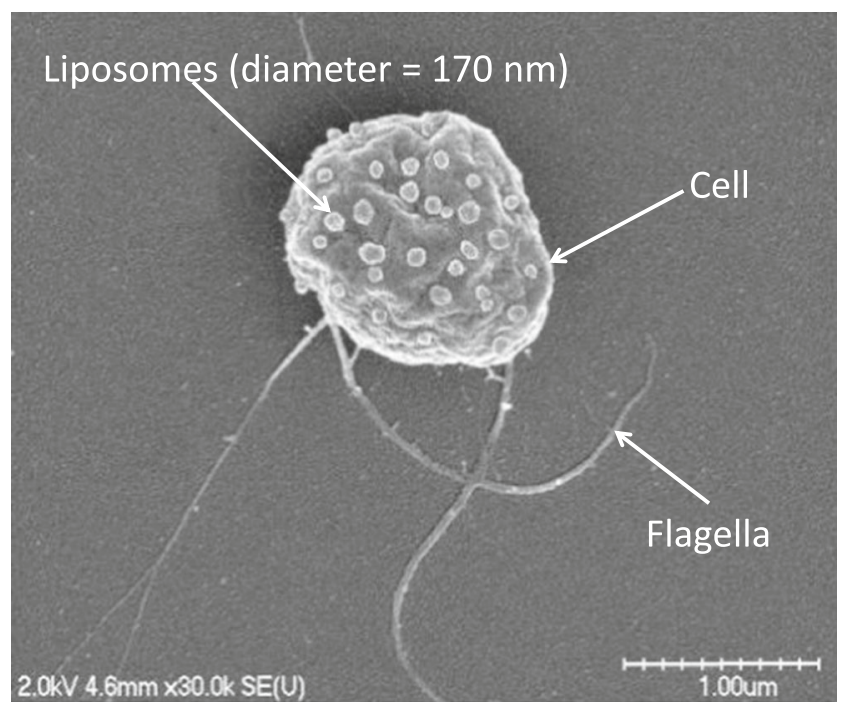

Fig. 1 Field-emission scanning electron microscopy image of one MC-1 MTB with approximately 70 liposomes attached to the surface of the cell
A large aggregation of these MTB can also be controlled in 3D [42] within an aqueous volume as depicted in Fig. 2. In this case, a special platform specially developed by our group for this purpose and referred to as the magnetotaxis system, was used. The magnetotaxis system is designed to create an "artificial magnetic pole" to force the MTB to migrate towards it. By using special modulating field patterns, such pole can be created anywhere within the operating space of the magnetotaxis system. When outside such a pole, a directional torque is induced on the chain of magnetosomes, forcing the MTB to swim towards such a pole. When inside the pole, the field intensity is insufficient to induce a directional torque. In this case, the MTB swim in a more random fashion within a zone which is referred to as the aggregation zone.

By maintaining the pole at the same location for a sufficient time, all MTB accumulate within the aggregation zone and can form a very large aggregation that can be observed without a microscope as depicted in Fig. 2. Once the aggregation is formed, it can be shifted to another position by varying the ratio of electrical currents in the coils of the magnetotaxis system.

With each bacterium loaded with a relatively large number of nano-components such as the example in Fig. 1, large computer-controlled bacterial transport and aggregation of nano-components within specific regions in a relatively large $3 \mathrm{D}$ aqueous workspace can be done.

\section{Power requirement}

One major advantage of this approach is the very low power requirement compared to other methods. Indeed, since the carrier is self-propelled, power is only required to induce a directional torque on the chain of magnetosomes in each cell.

The alignment of the magnetosomes creates a cell magnetic dipole that when exposed to a magnetic field can be described by the Langevin function as

$\cos \Theta=L\left(\frac{m \mu_{0} H}{k_{B} T}\right)$

where $\Theta$ is the angle between the direction of the cell magnetic moment $m$ and the ambient directional field $H=\mu_{0}^{-1} B$, $B$ being the magnetic field density and $\mu_{0}$ the permeability of free space. The Boltzmann constant and the temperature are represented by $k_{B}$ and $T$ respectively. Thus, the level of directional control characterized by the alignment of the cell in the applied directional magnetic field is determined by the ratio of the interactive magnetic energy with the applied field $(m B)$ to the thermal energy $\left(k_{B} T\right)$, thermal forces associated with Brownian motion that tend to randomize the cell orientation. At room temperature, it is still relatively easy technologically 
Fig. 2 MTB aggregation being moved in $3 \mathrm{D}$ by the magnetotaxis system. (Adapted from [42])
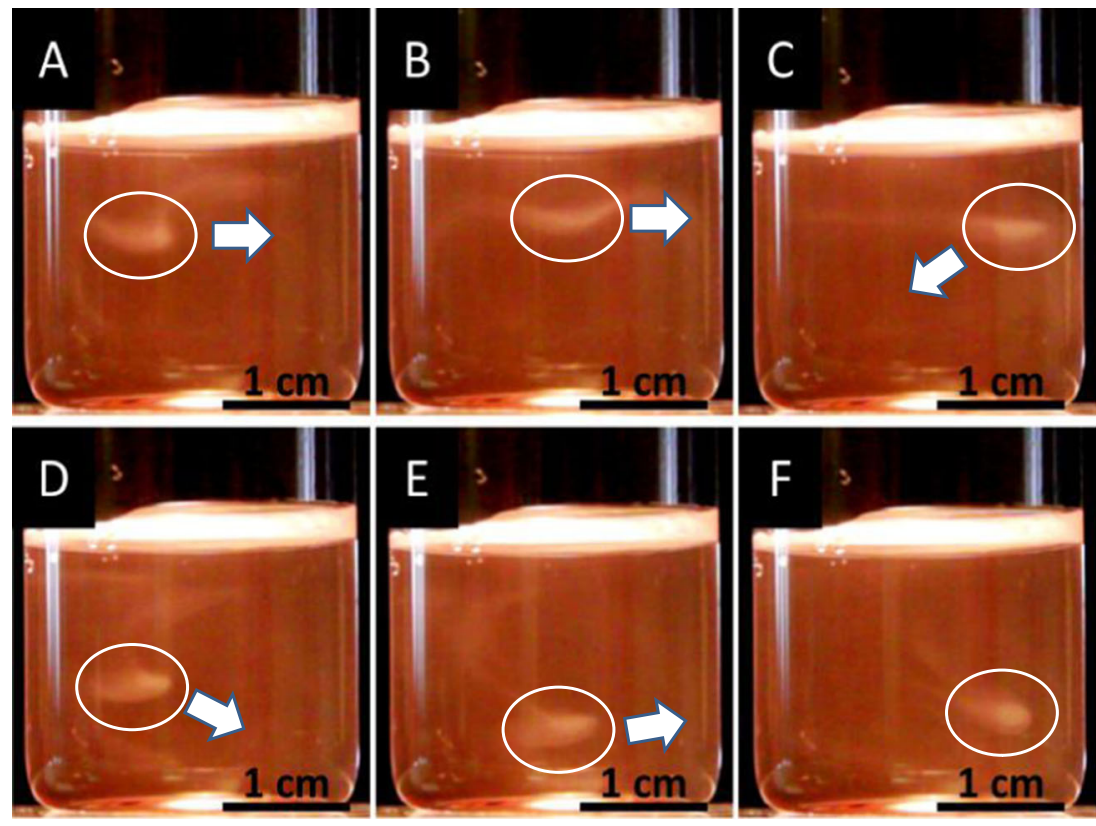

to apply over a relatively long distance (e.g. $0.5 \mathrm{~m}$ ) sufficiently high directional field to induce a torque on the chain of magnetosomes to align the cell along the magnetic field lines. But within a much smaller working volume as required in most cases, the power required would decrease exponentially. It was observed that when the ratio $m B k_{B} T \geq 10$, the cell gets fully aligned in the field direction with at least $90 \%$ efficiency. Considering that the total magnetic energy of the magnetosome chain takes into account the contribution of the 10 to 15 closely spaced individual magnetite $\left(\mathrm{Fe}_{3} \mathrm{O}_{4}\right)$ nanoparticles magnetic moment per MC-1 cell with $m=$ $10^{-16}\left(\mathrm{~A} \mathrm{~m}^{2}\right)$, a minimum magnetic field in the order of just a few Gauss (e.g. 4 Gauss) is sufficient to achieve proper magnetotactic directional control (90\% efficiency).

\section{MC-1 bacteria vs. artificial micro-swimmers}

Artificial versions of flagellated bacteria would offer constant velocities without any decay overtime. Indeed, various miniaturized helical micro-swimmers including Artificial Bacterial Flagella $(\mathrm{ABF})$ configurations have been investigated. $\mathrm{ABF}$ exploits the torque generated by a directional rotating magnetic field to move in a given direction. Although the magnetic field necessary for propulsion is reduced compared to using a magnetic pulling force, the resulting velocities are still far inferior to the ones achieved by the MC-1 MTB. The maximum velocity that was recorded was for a $38 \mu \mathrm{m}$ in length $\mathrm{ABF}$ was $18 \mu \mathrm{m} \mathrm{s}^{-1}$ using a rotating magnetic field of $2.0 \mathrm{mT}$ (maximum thrust (pushing) force of $3 \mathrm{pN}$ ) [43] resulting to approximately half a body length per second compared to the 100 to 150 cell lengths per second for the average and peak velocities respectively for the MC-1 cell. The structure of the $\mathrm{ABF}$ could be optimized to achieve superior relative velocities. For instance, a recent study showed that the velocity of this particular $\mathrm{ABF}$ could be increased to $170 \%$ of its original value [44] which is still modest compared to the MC-1 cell.

Measurement of the velocities of the MC-1 bacteria in Phosphate Buffer Saline (PBS) which had a viscosity equivalent to water at $20{ }^{\circ} \mathrm{C}$ (fluid viscosity $\eta_{F}=1 \times 10^{-3} \mathrm{~Pa} \cdot \mathrm{s}$ ) yielded an average velocity $\boldsymbol{v}=187.85 \mu \mathrm{m} \mathrm{s}^{-1}$. Assuming an average diameter of the spherical MC-1 cells of $1.5 \mu \mathrm{m}$ ( $\mathrm{min}$. and max. diam. of approx. 1 and $2 \mu \mathrm{m}$ respectively), the average thrust force is estimated in this particular case at $2.6 \mathrm{pN}$ which is equivalent to present versions of $\mathrm{ABF}$ having an overall size much larger than a single MC-1 cell. In term of power requirement, the rotating magnetic field of $20 \mathrm{mT}(20$ Gauss) is presently 5 times higher than the magnetic field required for inducing a directional torque on the MC-1 cells for achieving optimal directional control.

Although ABF can operate in more extreme fluidic environmental conditions that could impact the efficiency of the MC-1 cells, the fluidic characteristics could be modified to achieve superior results. For instance, bacteria typically show an increase in velocity in more viscous solutions up to a certain value $[45,46]$. Such increase can be explained by an alteration of the flagellar conformation by the external viscosity of the medium. Furthermore, it appears that the stators in bacterial cells act as dynamic mechanosensors and change their structure in response to changes in external load to gradually adapt by increasing their speeds in a stepwise manner [47]. This suggests that the extra-loads carried by the cell could be somewhat compensated by such mechanisms. But there is a limit for such compensation as preliminary 
experiments showed a slight decrease in the experiments where the MC-1 cells were loaded with $170 \mathrm{~nm}$ liposomes.

Another advantage of magnetotaxis-based self-propelled agents such as the MC-1 cells is the possibility of aggregate them in a specific region which may prove to be difficult with artificial agents.

\section{Loading density per cell}

Results from various instruments including the use of confocal microscopy revealed that the liposomes were firmly linked to the cell surface of the MC-1 MTB. Further experiments under harsh conditions showed that the same liposomes remained localized on the cell surface. More interesting is the fact that Scanning Electron Microscopy (SEM) images revealed that following a self-assembly approach where the MTB were simply mixed with the liposomes, that most liposomes were bound to the surface of MTB main body with less than $2 \%$ being attached to the flagella. Minimizing the latter is essential as it can affect the motility of the bacteria.

Optimizing the loading density per cell such as the number of liposomes in this particular case without compromising bacterial motility is a key objective. The present loading density was limited to the coupling of a maximum of 70 liposomes per cell. With an average size of $170 \mathrm{~nm}$, this result corresponds to approximately only $23 \%$ of the total cell surface. Since the bacterial motility and magnetotactic response were not significantly affected by such loading suggests that the loading density could be further increased. Although further experiments and measurements are required to confirm it, Fig. 1 could suggest the presence of a repulsive force between liposomes that could prevent further increase in the loading density per cell. Such observation and preliminary experimental results stress the need for further investigations in liposomal formulation or the use of other compositions such as biodegradable polymer-based nanoparticles encapsulating specific nano-components.

\section{Conclusions}

Bacteria can be effective carriers of nano-components. With various methods available, such nano-components can be attached to the surface of the cells. To achieve computercontrolled transport, magnetotactic bacteria have been considered. While displacement control can be achieved on a planar surface or microfluidic networks by inducing a directional torque on the chain of magnetosomes in each cell using a weak directional magnetic field, 3D displacement control is also possible using a special apparatus dubbed here magnetotaxis system. With an average of 70 nanocomponents being attached per magnetotactic bacterium, by converging magnetic field lines towards a specific location and using specific modulation sequences, a large quantity of nano-components can be transported to a specific location within a 3D space with minimum electrical energy requirement prior to be released through several possible methods including but not limited to change in $\mathrm{pH}$ and by biodegradation. Release mechanisms have not been investigated in this study and could be the topic of further investigations. Furthermore, although nanometer-size components have been considered in this paper, motility results obtained with liposomes suggests that an object of a few micrometers in diameter could be attached and transported by a single MC-1 bacterium.

Acknowledgments This project was partially funded by the Québec Consortium for Drug Discovery and a Research Chair in nanorobotics from Polytechnique Montréal.

\section{References}

1. Honda T, Arai KI, Ishiyama K (1996) Micro swimming mechanisms propelled by external magnetic fields. IEEE Trans Magn 32(5):50855087

2. Ghosh A, Fisher P (2009) Controlled propulsion of artificial magnetic nanostructured propellers. Nano Lett 9(6):2243-2245

3. Zhang L, Abbott JJ, Dong LX, Kratochvil BE, Bell DJ, Nelson BJ (2009) Artificial bacterial flagella: fabrication and magnetic control. Appl Phys Lett 94:064107

4. Zhang L, Petit T, Lu Y, Kratochvil BE, Peyer KE, Pei R, Lou J, Nelson BJ (2010) Controlled propulsion and cargo transport of rotating nickel nanowires near a patterned solid surface. ACS Nano 4(10):6228-6234

5. Zhang L, Abbott JJ, Dong L, Kratochvil BE, Zhang H, Peyer KE, Nelson BJ (2009) "Micromanipulation using artificial bacterial flagella." IEEE/RSJ Int. Conf. on Intelligent Robots and Systems, St. Louis, USA, pp 1401-1401

6. Zhang L, Peyer KE, Nelson BJ (2010) Artificial bacterial flagella for micromanipulation. Lab Chip 10:2203-2215

7. Berg HC, Turner L (1979) Movement of microorganisms in viscous environments. Nature 278:349-351

8. Greenberg EP, Canale-Parola E (1977) Motility of flagellated bacteria in viscous environments. J Bacteriol 132:356-358

9. Chen X, Berg HC (2000) Torque-speed relationship of the flagellar rotary motor in Escherichia coli. Biophys J 78:1036-1041

10. Chwang AT, Wu TY (1971) A note on the helical movement of micro-organisms. Proc R Soc Lond B 178:327-346

11. Holwill MEJ, Burge RE (1963) A hydrodynamic study of the motility of flagellated bacteria. Arch Biochem Biophys 101:249-260

12. Berg H (2004) E. coli in motion. Springer, New York

13. Magariyama Y, Sugiyama S, Kudo S (2001) Bacterial swimming speed and rotation rate of bundled flagella. FEMS Microbiol Lett 199:125-129

14. Magariyama Y, Sugiyama S, Muramoto K, Kawagishi I, Imae Y, Kudo S (1995) Simultaneous measurement of bacterial flagellar rotation rate and swimming speed. Biophys J 69:2154-2162

15. Shoesmith JG (1960) The measurement of bacterial motility. J Gen Microbiol 22:528-535

16. Martel S. "Controlled bacterial micro-actuation." In: Proc. of the Int. Conf. on Microtechnologies in Medicine and Biology, Okinawa, Japan, pp 89-92, May 9-12, 2006 
17. Martel S, Tremblay C, Ngakeng S, Langlois G (2006) Controlled manipulation and actuation of micro-objects with magnetotactic bacteria. Appl Phys Lett 89:233804-233806

18. Martel S, Mohammadi M. "Controllable bacterial actuators for nanorobots," 11th Int. Conf. on New Actuators (ACTUATOR) and 5th Int. Exhibition on Smart Actuators and Drive Systems, Bremen, Germany, June 9-11, 2008

19. Lu Z, Martel S. "Controlled bio-carriers based on magnetotactic bacteria," The 14th Int. Conf. on Solid-state Sensors and Actuators, Lyon, France, June 10-14, 2007

20. Martel S. "Magnetotactic bacteria as controlled functional carriers in microsystems, microelectronic circuits and interconnections," 16th European Microelectronics and Packaging Conference (EMPC), Oulu, Finland, June 17-20, 2007

21. Martel S, Mohammadi M. "Using a swarm of self-propelled natural microrobots in the form of flagellated bacteria to perform complex micro-assembly tasks.” In: Proc. of the IEEE Int. Conf. on Robotics and Automation (ICRA), Anchorage, Alaska, USA, May 3-8, 2010

22. Frankel RB, Blakemore RP (1980) Navigational compass in magnetic bacteria. J Magn Magn Mater 15-18(3):1562-1564

23. Debarros H, Esquivel DMS, Farina M (1990) Magnetotaxis. Sci Program 74:347-359

24. Frankel RB (1984) Magnetic guidance of organisms. Annu Rev Biophys Bioeng 13:85-103

25. Matsunaga T, Sakaguchi Y, Tadokoro F (1991) Magnetite formation by a magnetic bacterium capable of growing aerobically. Appl Microbiol Biotechnol 35:651-655

26. Sakaguchi T, Burgess JG, Matunaga T (1993) Magnetite formation by a sulphate reducing bacterium. Nature $365: 47-49$

27. Taylor BL, Koshland DE (1975) Intrinsic and extrinsic light responses of Salmonella typhimurium and Escherischia coli. J Bacteriol 123:557-569

28. Steager E, Kim C, Patel J, Bith S, Naik C, Reber L, Kim M (2007) Control of microfabricated stuctures powered by flagellated bacteria using phototaxis. Appl Phys Lett 90:263901-263903

29. Barak R, Nur I, Okon Y, Henis Y (1982) Aerotactic response of Azospirillum brasilense. J Bacteriol 152:643-649

30. Hou S, Larsen RW, Boudko D, Riley CW, Karatan E, Zimmer M, Ordal GW, Alam M (2000) Myoglobin-like aerotaxis transducers in archaea and bacteria. Nature 403:540-544

31. Baracchini O, Sherris JC (1959) The chemotactic effect of oxygen on bacteria. J Pathol Bacteriol 77:565
32. Adler J (1966) Chemotaxis in bacteria. Science 153:708-716

33. Berg HC, Brown DA (1972) Chemotaxis in Escherichia coli analysed by three-dimensional tracking. Nature 239:500-504

34. Schnitzer MJ, Block SM, Berg HC, Purcell EM (1990) Strategies for chemotaxis. Symp Soc Gen Microbiol 46:15-34

35. Behkam B, Sitti M (2007) Bacterial flagella-based propulsion and on/ off motion control of microscale objects. Appl Phys Lett 90:023902

36. Hermansson M (1999) The DLVO theory in microbial adhesion. Colloids Surf B: Biointerfaces 14:105-119

37. van Loosdrecht MCM, Lyklema J, Zehnder AJB (1989) Bacterial adhesion: a physicochemical approach. Microb Ecol 17:1-15

38. van Loosdrecht MCM, Lyklema J, Zehnder AJB (1990) Hydrophobic and electrostatic parameters in bacterial adhesion. Aquat Sci 52:103-114

39. Van Loosdrecht M, Lyklema J, Norde W, Schraa G, Zehnder A (1987) The role of bacterial cell wall hydrophobicity in adhesion. Appl Environ Microbiol 53:1893-1897

40. Tsuneda S, Aikawa H, Ayashi H, Yuasa A, Hirata A (2003) Extracellular polymeric substances responsible for bacterial adhesion onto solid surface. FEMS Microbiol Lett 223:287-292

41. Palomar J, Leranov A, Vinas M (1995) Serratia marcescens adherence: the effect of O-antigen presence. Microbios 81:107-113

42. de Lanauze D, Felfoul O, Turcot J-P, Mohammadi M, Martel S (2013) "Three-dimensional remote aggregation and steering of magnetotactic bacteria microrobots for drug delivery applications." Int J Robot Res 32(13)

43. Zhang L, Abbott JJ, Dong L, Peyer KE, Kratochvil BE, Zhang H, Bergeles C, Nelson BJ (2009) Characterizing the swimming properties of artificial bacterial flagella. Nano Lett 9(10):3663-3667

44. Keaveny EE, Walker SW, Shelley MJ (2013) Optimization of chiral structures for microscale propulsion. Nano Lett 13:531-537

45. Schneider WR, Doetsch RN (1974) Effect of viscosity on bacterial motility. J Bacteriol 117(2):696-701

46. Magariyama Y, Kudo S (2002) A mathematical explanation of an increase in bacterial swimming speed with viscosity in linearpolymer solutions. Biophys J 83:733-739

47. Lele PP, Hosu BG, Berg HC (2013) "Dynamics of mechanosensing in bacterial flagellar motor." PNAS, Early Edition, pp 1-6

48. Taherkhani S, Mohammadi M, Daoud J, Martel S, Tabrizian M. (2014) "Covalent binding of nanoliposomes to the surface of magnetotactic bacteria acting as self-propelled target delivery agents." ACS Nano, doi:10.1021/nn5011304 